13.4

\title{
Моделирование формирования цепочки виртуальных катодов в конической трубе дрейфа
}

\author{
(C) А.Е. Дубинов ${ }^{1,2}$, В.П. Тараканов ${ }^{3,4}$, \\ ${ }^{1}$ Российский федеральный ядерный центр - Всероссийский научно-исследовательский институт экспериментальной \\ физики, Саров, Нижегородская обл., Россия \\ ${ }^{2}$ Саровский физико-технический институт, Саров, Нижегородская обл., Россия \\ ${ }^{3}$ Объединенный институт высоких температур РАН, Москва, Россия \\ ${ }^{4}$ Национальный исследовательский ядерный университет „МИФИ“, Москва, Россия \\ E-mail: dubinov-ae@yandex.ru
}

Поступило в Редакцию 26 марта 2019г.

В окончательной редакции 15 апреля 2019г.

Принято к публикации 16 апреля 2019г.

С помощью РІС-моделирования показано, что в конической трубе дрейфа с увеличивающимся по ходу движения электронного пучка диаметром возникает цепочка из нескольких виртуальных катодов. Рассчитана их пространственно-временна́я динамика.

Ключевые слова: электронный пучок, труба дрейфа, конус, виртуальный катод.

DOI: 10.21883/PJTF.2019.15.48079.17805

Известно, что, если в замкнутую эквипотенциальную полость инжектировать электронный пучок с током, превышающим значение предельного вакуумного тока, в полости возникает так называемый виртуальный катод (ВК) [1]. Обычно если пучок один, то и ВК один. Электровакуумные приборы с ВК получили свое развитие при создании сильноточных ускорителей ионов $[2,3]$ и являются основой мощных СВЧ-генераторов - виркаторов [4-8].

Существует задача поиска условий формирования в одном пучке сразу нескольких ВК. Это позволило бы, например, более эффективно отбирать энергию электронного пучка для СВЧ-излучения.

В настоящей работе с помощью particle-in-cell-моделирования (РІС-моделирования) показано, что в замагниченной полости, имеющей коническую форму, с увеличивающимся по ходу движения электронного пучка диаметром может возникать цепочка сразу из нескольких ВК.

Сначала поясним, в чем особенность именно конической трубы дрейфа.

Термин „предельный вакуумный ток“ сильноточного электронного пучка в эквипотенциальной полости дрейфа является ключевым понятием в сильноточной вакуумной электронике [9], в том числе и для СВЧ-генераторов с ВК. Известно, например, что если в полость инжектировать электронный пучок с током, превышающим значение предельного вакуумного тока, то там возникнет ВК, отражающий часть электронов пучка назад, к месту его инжекции. При этом ток неотраженной части пучка в среднем за период колебаний будет равен предельному вакуумному току $[10,11]$.

Общепринято, что понятие ,предельный вакуумный ток“ относится к полости дрейфа целиком и опре- деляет ее пропускную способность для электронного пучка $[12,13]$. Величина предельного вакуумного тока зависит от геометрических параметров полости, геометрических параметров пучка, энергии инжектированных электронов, а также от величины и параметров геометрии магнитного поля.

Если рассматривать длинные трубы дрейфа с поперечным размером, много меньшим ее длины, то понятия „предельный вакуумный ток“, относящегося ко всей трубе дрейфа, для некоторых задач недостаточно. По нашему мнению, в ряде случаев целесообразно ввести понятие „локальный предельный вакуумный ток“, относящееся к каждому конкретному поперечному сечению трубы дрейфа.

В самом деле, если рассматривать, например, замагниченную длинную трубу дрейфа круглого сечения переменного диаметра, можно предположить, что на участке с меньшим диаметром локальный предельный ток и, следовательно, пропускная способность трубы окажутся бо́льшими, чем на участке с бо́льшим диаметром. Любопытно, что это правило прямо противоположно правилу о пропускной способности обычного трубного водопровода, где чем больше диаметр трубы, тем больше ее локальная пропускная способность для воды.

Представим себе, что пучок инжектируется в трубу дрейфа круглого поперечного сечения с монотонно растущим по ходу движения электронов диаметром. Локальный предельный ток по ходу движения должен монотонно убывать. Тогда на некотором небольшом расстоянии от того сечения трубы, в котором значение локального предельного тока станет меньше тока пучка, сформируется ВК, который отразит часть электронов назад. Ток пролетевшей ВК части пучка в соответствии 


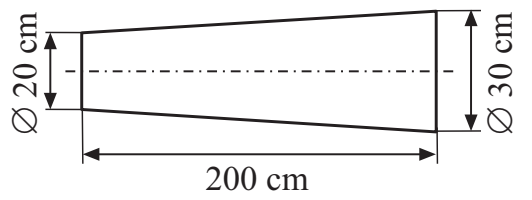

Рис. 1. Геометрия конической трубы дрейфа.

c $[10,11]$ будет равен локальному предельному току в рассматриваемом сечении.

Далее, на некотором небольшом расстоянии по ходу движения пучка полетный ток пучка снова превысит локальный предельный ток и создадутся условия для формирования второго ВК и т.д. В итоге, продолжая подобные рассуждения снова и снова, приходим к выводу, что в трубе дрейфа с монотонно растущим по ходу движения электронов диаметром должна сформироваться цепочка ВК. При этом ВК должны отстоять друг от друга на расстояние, не меньшее необходимого для торможения сильноточного электронного пучка (СЭП) до полной остановки электронов. Такая уникальная ситуация с цепочкой ВК, которая возможна в расширяющейся по ходу движения электронов трубе дрейфа, как видно, легко объясняется с помощью понятия локального предельного тока.

Подчеркнем, что режимы, в которых в трубе дрейфа с уменьшающимся по ходу движения электронов локальным предельным током могла бы сформироваться цепочка из нескольких ВК, ранее нигде не рассматривались, и поэтому они требуют подтверждения и исследования на динамических моделях и в экспериментах.

Динамика формирования ВК в простейшей по геометрии трубе дрейфа с убывающим локальным предельным током - конической трубе дрейфа - исследована в настоящей работе. Для этого проведено самосогласованное моделирование с помощью РІС-кода „КАРАТ“ (версия 2.5D) [14].

„КАРАТ“ представляет собой электромагнитный код на базе РІС-метода. Он предназначен для решения нестационарных электродинамических задач, имеющих сложную геометрию и включающих динамику в общем случае релятивистских частиц (электронов, ионов, нейтралов).

Для решения уравнений Максвелла применяется разностная схема с перешагиванием на прямоугольных сетках со сдвигом. Конкретная реализация схемы, примененная в коде, обладает свойством точного описания граничных условий на поверхностях расчетной области.

Укажем и на другие современные коды, в которых возможно аналогичное РІС-моделирование приборов с BK („MAGIC“ $[15,16]$, „CST Particle Studio“ $[16,17])$.

Отметим, что недавно виркатор с конической трубой дрейфа исследовался с помощью кода „КАРАТ“ в [18], однако режимы с формированием нескольких ВК там не рассматривались.
Для исследования формирования цепочки ВК в настоящей работе моделировалась динамика пучка, инжектируемого в трубу дрейфа с увеличивающимся по ходу движения электронов диаметром. Задавалась находящаяся в сильном однородном аксиальном магнитном поле и замкнутая с торцов прозрачными для
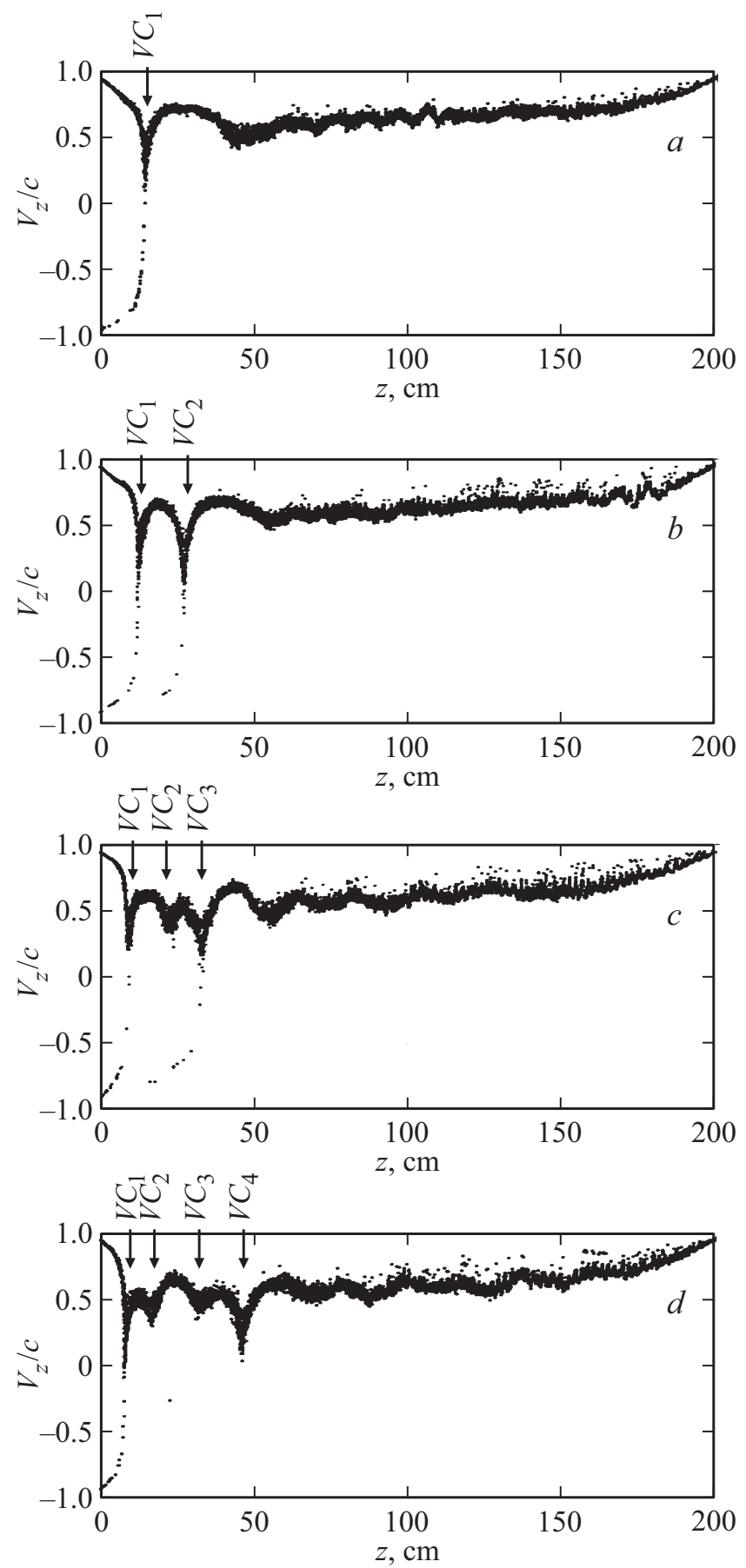

Рис. 2. Последовательность мгновенных фазовых портретов СЭП в конической трубе дрейфа. $V C_{i}-i$-й ВК. $a-8.5 \mathrm{~ns}$ (сформировался только $\left.V C_{1}\right), b-15.0 \mathrm{~ns}$ (сформировались $V C_{1}$ и $V C_{2}$ ), $c-22.5 \mathrm{~ns}$ (сформировались $V C_{1}$ и $V C_{3}$, а $V C_{2}$ исчезает), $d-25.5 \mathrm{~ns}$ (существует $V C_{1}$, исчезают $V C_{2}$ и $V C_{3}$, рождается $\left.V C_{4}\right)$. 


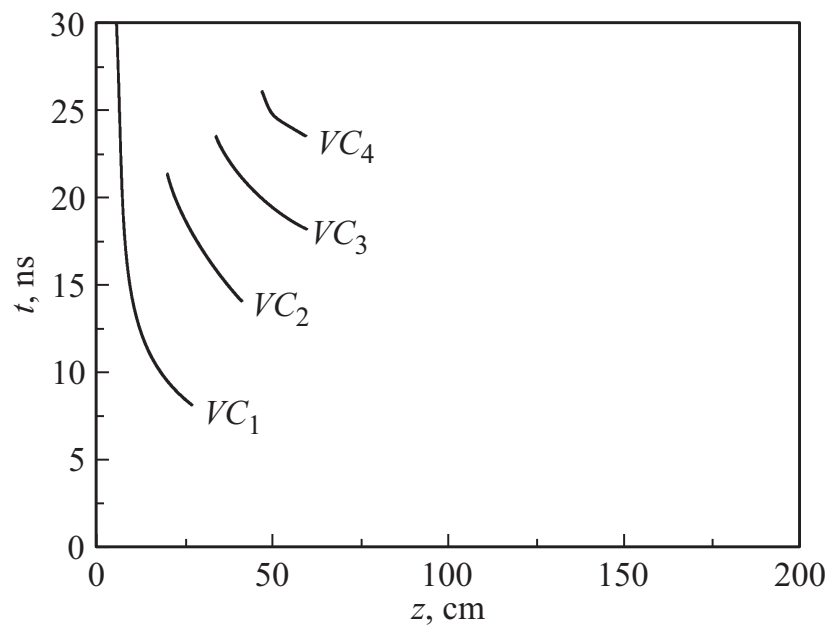

Pис. 3. Пространственно-временна́я динамика цепочки ВК.

электронов проводящими фольгами коническая труба дрейфа (рис. 1). Считалось, что инжекция трубчатого СЭП производится со стороны узкого сечения трубы (на рис. 1 слева) с постоянным током. Было принято, что электроны СЭП в плоскости инжекции имеют одну и ту же энергию $(1000 \mathrm{keV})$. Внешний диаметр СЭП составляет $8 \mathrm{~cm}$, толщина трубки тока СЭП $-1 \mathrm{~cm}$.

На рис. 2 показана последовательность мгновенных фазовых портретов СЭП при токе инжекции $9 \mathrm{kA}$. Оказалось, что в конической трубе дрейфа действительно формируется цепочка из нескольких ВК, однако ВК рождаются не одновременно, и время существования каждого ВК, кроме первого, конечно.

Происходит это так. Сначала формируется первый ВК (обозначен на рис. 2 как $V C_{1}$ ), который располагается ближе всех к месту инжекции СЭП. Затем через несколько наносекунд формируется второй ВК $-V C_{2}$. В течение следующих нескольких наносекунд $V C_{2}$ исчезает, но рождается $V C_{3}$. Еще через несколько наносекунд $V C_{3}$ исчезает и рождается $V C_{4}$ и т.д. При этом $V C_{1}$ существует всегда, пока инжектируется СЭП.

Следует отметить, что исчезновение некоторых ВК происходит не бесследно: на их месте в течение длительного времени остаются локальные минимумы потенциала и совпадающие с ними максимумы плотности электронов. Эти „остатки“ ВК в виде фазовых электронных сгустков продолжают осциллировать вдоль оси трубы дрейфа и участвовать в генерации электромагнитного излучения.

По результатам моделирования была восстановлена пространственно-временна́я динамика ВК. Она показана на рис. 3. Оказалось, что все ВК формируются только в первой половине трубы дрейфа.

Такое поведение пучка в конической трубе дрейфа со множеством ВК принципиально отличается от поведения пучка в цилиндрической трубе дрейфа, в которой ВК всегда единствен. Найденный в работе режим мо- жет оказаться полезным для получения эффективной СВЧ-генерации в виркаторах.

\section{Конфликт интересов}

Авторы заявляют, что у них нет конфликта интересов.

\section{Список литературы}

[1] Миллер Р. Введение в физику сильноточных пучков заряженных частиц. М.: Мир, 1984. 432 с.

[2] Быстрицкий В.М., Диденко А.Н. // УФН. 1980. Т. 132. № 9. C. 91-122.

[3] Дубинов А.Е., Корнилова И.Ю., Селемир В.Д. // УФН. 2002. T. 172. № 11. C. 1225-1246.

[4] Hoeberling R.F., Fazio M.V. // IEEE Trans. Electromagn. Compat. 1992. V. 34. N 3. Pt 1. P. 252-258.

[5] Рухадзе А.А., Столбецов С.Д., Тараканов В.П. // Радиотехника и электроника. 1992. Т. 37. № 3. С. 385-396.

[6] Дубинов А.Е., Селемир В.Д. // Радиотехника и электроника. 2002. Т. 47. № 6. С. 645-672.

[7] Alyokhin B.V., Dubinov A.E., Selemir V.D., Shamro O.A., Shibalko K.V., Stepanov N.V., Vatrunin V.E. // IEEE Trans. Plasma Sci. 1994. V. 22. N 5. P. 945-959.

[8] Селемир В.Д., Дубинов А.Е., Птицын Б.Г., Евсеенко А.А., Летягин В.А., Нургалиев Р.К., Судовцов А.В., Ячный А.В. // ЖТФ. 2001. Т. 71. В. 11. С. 68-72.

[9] Богданкевич Л.С., Рухадзе А.А. // УФН. 1971. Т. 103. № 4. C. 609-640.

[10] Seo Y., Choi E.H., Byun C.G., Choi M.C. // Jpn. J. Appl. Phys. 2001. V. 40. N 2B. Pt 1. P. $1136-1139$.

[11] Дубинов А.Е., Ефимова И.А. // ЖТФ. 2003. Т. 73. В. 9. C. 126-129.

[12] Альтеркоп Б.А., Сокулин А.Ю., Тараканов В.П. // Физика плазмы. 1989. Т. 15. № 8. С. 974-980.

[13] Сотников Г.В., Яценко Т.Ю. // ЖТФ. 2002. Т. 72. В. 5. C. $22-25$.

[14] Tarakanov V.P. User's manual for code KARAT. Springfield: Berkley Research Associates, 1992. $127 \mathrm{p}$.

[15] Mumtaz S., Lim J.S., Ghimire B., Lee S.W., Choi J.J., Choi E.H. // Phys. Plasmas. 2018. V. 25. N 10. P. 103113.

[16] Champeaux S., Gouard P., Cousin R., Larour J. // IEEE Trans. Plasma Sci. 2015. V. 43. N 11. P. 3841-3855.

[17] Dubinov A.E., Petrik A.G., Kurkin S.A., Frolov N.S., Koronovskii A.A., Hramov A.E. // Phys. Plasmas. 2017. V. 24. N 7. P. 073102.

[18] Dubinov A.E., Tarakanov V.P. // IEEE Trans. Plasma Sci. 2016. V. 44. N 8. Pt 2. P. 1391-1395. 